\title{
Feasibility of Therapeutic Drug Monitoring of Sunitinib and its implications on response and toxicity in patients with metastatic renal cell cancer
}

\author{
Khushboo Gandhi ${ }^{1}$, Amit Joshi ${ }^{2}$, Parsshava Mehta ${ }^{1}$, Murari Gurjar ${ }^{1}$, Pallavi Rane ${ }^{1}$, Jyoti \\ Sharma ${ }^{1}$, Anand Patil ${ }^{1}$, Manjunath Nookala ${ }^{1}$, Vanita Noronha ${ }^{2}$, Kumar Prabhash ${ }^{2}$, and \\ Vikram Gota ${ }^{1}$ \\ ${ }^{1}$ Advanced Centre for Treatment Research and Education in Cancer \\ ${ }^{2}$ Tata Memorial Hospital
}

November 8, 2021

\begin{abstract}
High interindividual variability in pharmacokinetics coupled with concentration-effect relationship make sunitinib an ideal candidate for therapeutic drug monitoring (TDM). The feasibility of TDM of sunitinib in patients with metastatic renal cell carcinoma $(\mathrm{mRCC})$ was evaluated in this prospective observational study. Seventy patients with mRCC treated with sunitinib 50mg OD were enrolled. Total trough levels (TTL) of sunitinib and N-desethyl sunitinib were measured between days 10-14 of cycle 1. The discriminatory potential of TTL of sunitinib for the prediction of responders and occurrence of grade [?]3 toxicity was determined using receiver operating characteristic (ROC) curve. Threshold concentrations obtained from ROC analysis showed that TTL [?]60.75ng/mL was associated with higher response rates and TTL [?]82.3ng/mL was associated with higher incidence of grade [?] 3 toxicity compared with lower exposures (31/34 versus $5 / 12, \mathrm{P}=0.001$ and $9 / 24$ versus $4 / 36$; $\mathrm{P}=0.024$ respectively). More than $50 \%$ of patients in our cohort attained TTL outside the optimal range of $60.75-82.3 \mathrm{ng} / \mathrm{mL}$ demonstrating the feasibility of TDM.
\end{abstract}

Title Page

Title: Feasibility of Therapeutic Drug Monitoring of Sunitinib and its implications on response and toxicity in patients with metastatic renal cell cancer.

Short Title: Feasibility of sunitinib TDM in renal cancer

Khushboo Gandhi ${ }^{\mathrm{a}, 1}$, Amit Joshic, d,1, Parsshava Mehta ${ }^{\mathrm{a}}$, Murari Gurjar ${ }^{\mathrm{a}}$, Pallavi Rane ${ }^{\mathrm{b}}$, Jyoti Sharma ${ }^{\mathrm{a}}$, Anand Patil ${ }^{\mathrm{a}, \mathrm{d}}$, Manjunath Nookala ${ }^{\mathrm{a}, \mathrm{d}}$, Vanita Noronha ${ }^{\mathrm{c}, \mathrm{d}}$, Kumar Prabhash ${ }^{\mathrm{c}, \mathrm{d}}$, Vikram Gota ${ }^{* a, d}$

1: Authors contributed equally to the present work

${ }^{a}$ Department of Clinical Pharmacology, Advanced Centre for Treatment, Research and Education in Cancer (ACTREC), Tata Memorial Centre (TMC), Kharghar, Navi Mumbai-410210, India.

${ }^{\mathrm{b}}$ Department of Biostatistics, Advanced Centre for Treatment, Research and Education in Cancer (ACTREC), Tata Memorial Centre (TMC), Kharghar, Navi Mumbai-410210, India.

${ }^{\mathrm{c}}$ Department of Medical Oncology, Tata Memorial Hospital, Parel, Mumbai-400012, India.

${ }^{\mathrm{d}}$ Homi bhabha National Institute, Mumbai-400094, India 
*To whom correspondence should be addressed: Dr. Vikram Gota, Advanced Centre for Treatment, Research and Education in Cancer (ACTREC), Tata Memorial Centre (TMC), Sector-22, Kharghar, Navi Mumbai410210, India, Tel.: +91-22-27405493; Fax: +91-22-27405085; E-mail: vgota76@actrec.gov.in

Principal Investigator statement : The authors confirm that the Principal Investigator for this paper is Dr. Vikram Gota and that he had direct clinical responsibility for patients.

Key Words: Sunitinib; Therapeutic Drug Monitoring; Objective response; SU12662; Toxicity

Word count of text: 1727

Table Count: 1

Figure Count: 2

\title{
What is already known about this subject:
}

High interindividual variability in pharmacokinetics coupled with a clear exposure-effect relationship make sunitinib an ideal candidate for therapeutic drug monitoring (TDM).

However, there are no recommendations for the TDM of sunitinib and hence not followed

What this study adds:

- The feasibility of TDM of sunitinib in patients with metastatic renal cell carcinoma (mRCC) was evaluated

- Optimal steady-state trough concentration of sunitinib was found to lie between $60.75-82.3 \mathrm{ng} / \mathrm{ml}$

- $>50 \%$ of patients had concentrations outside the optimal range, hence TDM is likely to improve safety and efficacy of sunitinib in mRCC

\begin{abstract}
:
High interindividual variability in pharmacokinetics coupled with concentration-effect relationship make sunitinib an ideal candidate for therapeutic drug monitoring (TDM). The feasibility of TDM of sunitinib in patients with metastatic renal cell carcinoma $(\mathrm{mRCC})$ was evaluated in this prospective observational study. Seventy patients with mRCC treated with sunitinib 50mg OD were enrolled. Total trough levels (TTL) of sunitinib and N-desethyl sunitinib were measured between days 10-14 of cycle 1 . The discriminatory potential of TTL of sunitinib for the prediction of responders and occurrence of grade [?]3 toxicity was determined using receiver operating characteristic (ROC) curve. Threshold concentrations obtained from ROC analysis showed that TTL [?] $60.75 \mathrm{ng} / \mathrm{mL}$ was associated with higher response rates and TTL [?] $82.3 \mathrm{ng} / \mathrm{mL}$ was associated with higher incidence of grade [?]3 toxicity compared with lower exposures $(31 / 34$ versus $5 / 12$, $\mathrm{P}=0.001$ and $9 / 24$ versus $4 / 36 ; \mathrm{P}=0.024$ respectively). More than $50 \%$ of patients in our cohort attained TTL outside the optimal range of $60.75-82.3 \mathrm{ng} / \mathrm{mL}$ demonstrating the feasibility of TDM.
\end{abstract}

\section{Introduction:}

Sunitinib malate was the standard first-line treatment for metastatic renal cell carcinoma (mRCC) globally till recently. Even today, in several low and middle income countries where immunotherapies are ill-afforded, it continues to be the standard of care. The pharmacokinetics of sunitinib is highly variable with a reported inter-patient coefficient of variation (CV) of up to $60 \%$ for various pharmacokinetic parameters ${ }^{1}$. Moreover, there is an excellent concentration-efficacy/toxicity relationship seen with sunitinib in patients of $\mathrm{mRCC}^{2,3}$. For these reasons, sunitinib is an ideal candidate for therapeutic drug monitoring (TDM) rather than using a fixed dose of $50 \mathrm{mg}$ that is currently in vogue. Maintaining a total target trough plasma concentration of sunitinib and its active metabolite (N-desethyl sunitinib, SU12662) in the range of 50-100 ng/mL has been proposed based on several clinical and non-clinical studies ${ }^{2,3,4}$. Higher sunitinib exposure is associated with an increased risk of severe adverse events, indicating a narrow therapeutic index. The threshold value of plasma composite (sunitinib + SU12662) exposure predicting grade 3-4 toxicities remains to be determined in routine clinical practice. In a retrospective study involving 59 patients, $23(39 \%)$ patients required dose 
interruption or dose delay while $12(20 \%)$ patients required dose modification due to toxicity ${ }^{5}$. Recent studies have indicated that PK guided dosing can optimize sunitinib therapy compared to standard fixed dose and can decrease the frequency of dose interruption. This study was planned to evaluate the feasibility of TDM of Sunitinib in patients with $\mathrm{mRCC}$ in a real world setting.

\section{Material \& Methods:}

The prospective observational study was conducted at Tata Memorial Centre, Mumbai. The study was approved by the Institutional Ethics Committee of Tata hospital. All trial participants provided written informed consent prior to their enrolment. The study was carried out in accordance with the Declaration of Helsinki and International Conference on Harmonization-Good Clinical Practice (ICH-GCP) guidelines. A steady state trough plasma sample was collected between days 10-14 of the first cycle. Sunitinib and SU12662 concentrations were measured simultaneously using ultra-high-performance liquid chromatography as described previously ${ }^{6}$. Sunitinib administration was continued until disease progression, intolerable adverse events or patient's refusal to take the drug for any reason. All adverse events were graded according to Common Terminology Criteria for Adverse Effects (CTCAE) version 4.03. All response assessments, until disease progression, were collated and the best response was determined as either complete response (CR), partial response (PR), stable disease (SD) or progressive disease (PD) as per RECIST $1.1^{7}$. The optimal cut-off value of sunitinib concentration for the prediction of the response and the occurrence of grade[?]3 toxicity was identified using the receiver operating characteristic (ROC) curve. Time to event endpoints were analysed using Kaplan-Meier plots and compared between groups using log-rank test. All computations were performed with the SPSS Version 25.0.

\section{Result and Discussion:}

Seventy patients aged 18 years or older with histologically or cytologically confirmed mRCC receiving sunitinib at $50 \mathrm{mg}$ dose daily were enrolled in the study. Recently the RESTORE trial, a phase II randomized trial compared the 4 -week on/2-week off $(4 / 2)$ schedule vs a 2 -week on/1-week off $(2 / 1)$ schedule. This study concluded that the administered $2 / 1$ schedule is associated with less toxicity and higher failure-free survival (FFS) at 6 months than the $4 / 2$ schedule, without compromising the efficacy in terms of objective response rate $(\mathrm{ORR})$ and time to progression $(\mathrm{TTP})^{8}$. Sixteen patients received sunitinib for $4 / 2$ regimen in a six week cycle and 54 patients received $2 / 1$ regimen.

The patient characteristics are summarized in table 1. The number of patients included in each analysis is summarized in Supplementary fig 1. Total trough levels of sunitinib (sunitinib + SU12662) (TTL) was determined for 60 out of 70 patients. The median TTL, trough sunitinib and SU12662 concentrations were 75.2 (24.5-209.4), 56.5 (16.5-129.4) and 19.8 (6.9-48.4) ng/mL respectively. The TTL of sunitinib was highly variable $(\mathrm{CV}=46.3 \%)$ (Supplementary fig 2$)$.

A total of 60 patients were evaluable for the analysis of the association between TTL of sunitinib and toxicity whereas 46 of the 70 patients were evaluable for the analysis of the association between TTL of sunitinib and response. During cycle 1 of treatment, toxicity of any grade was observed in 50 out of 60 patients (83.3\%). Grade [?]3 toxicity were observed in 12 patients. Grade [?]3 toxicities attributed to sunitinib treatment included hypertension, fatigue, hyponatremia, thrombocytopenia and hand-foot syndrome (HFS). The ability of TTL to predict grade [?]3 toxicity and response (having SD or better as best response) is shown in the ROC curve analysis in Figure $1 \mathrm{~A}$ and $1 \mathrm{~B}$ respectively. TTL of $82.3 \mathrm{ng} / \mathrm{mL}$ was found to be discriminatory for the prediction of grade [?]3 toxicity (AUC: 0.678, 95\% CI: 0.516-0.839, Sensitivity: 69.23\%, Specificity: $68.09 \%, \mathrm{p}=0.05)$ whereas TTL of $60.75 \mathrm{ng} / \mathrm{mL}$ was discriminatory for the prediction of responders (AUC: 0.797, 95\% CI: 0.618-0.977, Sensitivity: $86.11 \%$, Specificity: $70 \%, \mathrm{p}=0.004$ ). The best overall response for all the patients is presented in supplementary figure 3 . The incidence of grade [?]3 toxicity was observed in $4 / 36(11.1 \%)$ patients in the low-exposure group (TTL $<82.3 \mathrm{ng} / \mathrm{mL})$ compared with $9 / 24(37.7 \%)$ patients in the high-exposure group (TTL [?] $82.3 \mathrm{ng} / \mathrm{mL})(\mathrm{p}=0.024)$. On the other hand an objective response was observed in only $5 / 12$ patients $(41.6 \%)$ in the low-exposure group (TTL $<60.75 \mathrm{ng} / \mathrm{mL}$ ) compared with $31 / 34$ patients $(91.1 \%)$ in the high-exposure group (TTL [?]60.75ng/mL) $(\mathrm{p}=0.001)$. 
On similar lines, analysis of association of toxicity/ response and TTL of sunitinib was done for 54 patients who received the $2 / 1$ regimen of sunitinib. Forty six patients were included in this analysis of toxicity whereas 36 were included in analysis of response (Supplementary fig 1). The ROC curve showed that the concentration of $82.3 \mathrm{ng} / \mathrm{mL}$ and $60.75 \mathrm{ng} / \mathrm{mL}$ was discriminatory for the prediction of grade [?]3 toxicity (AUC: $0.688,95 \%$ CI: 0.435-0.940, Sensitivity: $83.33 \%$, Specificity: $72.50 \%, \mathrm{p}=0.142$ ) and responders (AUC: 0.818, 95\% CI: 0.746-1.000, Sensitivity: $85.19 \%$, Specificity: $88.89 \%$ and p: 0.001 ) respectively (Figure $2 \mathrm{~A}$ \& 2B). The incidence of grade[?]3 toxicity was observed in 1/30 (3.33\%) patients in the low-exposure group (TTL $<82.3 \mathrm{ng} / \mathrm{mL}$ ) compared with $5 / 16$ patients $(31.25 \%)$ in the high-exposure group (TTL [?]82.3ng/mL) $(\mathrm{p}=0.015)$. An objective response was observed in $4 / 12$ patients $(33.3 \%)$ in the low-exposure group (TTL $<60.75 \mathrm{ng} / \mathrm{mL}$ compared with $23 / 24$ patients $(95.8 \%$ ) in the high-exposure group (TTL [?]60.75ng/mL) $(\mathrm{p}<0.001)$.

The median follow-up for all patients was 29.2 (22-34.4) months while it was 29 (18-40) months for the patients receiving $2 / 1$ regimen. The probability of 2 years survival was $13.4 \%$ in the low-exposure group (TTL $<60.75 \mathrm{ng} / \mathrm{mL}$ ) as compared to $38.8 \%$ in the high-exposure group (TTL [?] $60.75 \mathrm{ng} / \mathrm{mL}$ ) for all patients (Figure 1C), whereas, for patients receiving $2 / 1$ dose regimen of sunitinib the probability of 2 years survival was $7 \%$ in the low-exposure group as compared to $40.8 \%$ in the high-exposure group (Figure $2 \mathrm{C}$ ).

Previous PK/PD data in a mouse xenograft model and phase I trials indicate that optimal target range for TTL is $50-100 \mathrm{ng} / \mathrm{mL}^{2,3,4}$. Previously Noda et al. has shown that dose reduction is needed, when the total sunitinib concentration is $>100 \mathrm{ng} / \mathrm{mL}$, to avoid unnecessary early discontinuation of treatment ${ }^{9}$. Uemura et al also have shown that sunitinib was effective at TTL of $50 \mathrm{ng} / \mathrm{mL}$ in patients with $\mathrm{mRCC}^{10}$. Our findings corroborate these observations albeit at slightly different threshold concentrations, indicating a TTL range of $60.75-82.3 \mathrm{ng} / \mathrm{ml}$ is ideal for optimal therapeutic effect. However, it may not be possible to maintain trough concentration within this narrow range, particularly through fixed oral dosing. Therefore, for practical reasons, the TTL range of $60-100 \mathrm{ng} / \mathrm{mL}$ may be most appropriate for our setting considering treatment failure rates increased dramatically in patients who did not achieve TTL of $60.75 \mathrm{ng} / \mathrm{mL}$.

It has been reported that severe sunitinib-induced acute toxicities can impact the quality of life (QoL) and lead to early treatment discontinuation ${ }^{11}$. Collecting longitudinal QOL data would have given better understanding of the state of health leading to continuation or discontinuation of sunitinib at various stages of treatment. It would have allowed us to study the association between drug levels and QoL and could have complimented the strategy for optimal dosing of sunitinib in mRCC.

To conclude, although scattered evidence favors exposure-monitored dosing, TDM has not been adopted into routine practice because of the absence of high level evidence in the form of large randomized controlled trials or systematic reviews. Here, we could show that patients with mRCC may benefit from TDM based dosing, and we were able to ascertain the therapeutic range in our population. A randomized trial of TDM based dosing versus conventional dosing is underway at our centre to conclusively establish the role of TDM in improving the safety and efficacy of sunitinib in mRCC.

\section{Acknowledgement:}

The authors gratefully acknowledge the Indian Council of Medical Research for partially funding this study through the Centre for Advanced Research and Excellence (ICMR-CARE) in Clinical Pharmacology (Grant No. 55/4/13/CARE-CP/2018-NCD-II). The authors acknowledge Mr. Sharath Kumar HJ for extending his help in making figures.

\section{Conflict of interest statement .}

None

\section{References}

1. Demlová R, Turjap M, Peš O, Kostolanská K, Juřica J. Therapeutic Drug Monitoring of Sunitinib in Gastrointestinal Stromal Tumors and Metastatic Renal Cell Carcinoma in Adults-A Review. Ther 
Drug Monit. 2020;42(1):20-32. doi:10.1097/FTD.0000000000000663. PMID: 31259881.

2. Houk BE, Bello CL, Poland B, Rosen LS, Demetri GD, Motzer RJ. Relationship between exposure to sunitinib and efficacy and tolerability endpoints in patients with cancer: results of a pharmacokinetic/pharmacodynamic meta-analysis. Cancer Chemother Pharmacol. 2010;66(2):357-371. doi:10.1007/s00280-009-1170-y. PMID: 19967539.

3. Faivre S, Delbaldo C, Vera K, et al. Safety, pharmacokinetic, and antitumor activity of SU11248, a novel oral multitarget tyrosine kinase inhibitor, in patients with cancer. J Clin Oncol. 2006;24(1):25-35. doi:10.1200/JCO.2005.02.2194

4. Mendel DB, Laird AD, Xin X, et al. In vivo antitumor activity of SU11248, a novel tyrosine kinase inhibitor targeting vascular endothelial growth factor and platelet-derived growth factor receptors: determination of a pharmacokinetic/pharmacodynamic relationship. Clin Cancer Res. 2003;9(1):327337. PMID: 12538485.

5. Krishna VM, Noronha V, Prabhash K, et al. Sunitinib in metastatic renal cell carcimoma: a single-center experience. Indian J Cancer. 2013;50(3):268-273. doi:10.4103/0019-509X.118725. PMID: 24061470

6. Gurjar M, Mehta P, Sharma J, Patil S, Kulkarni P, Patil A, Nookala KM, Joshi A, Gota V. An HPLC method for simultaneous quantification of sunitinib and its active metabolite, SU12662, using hydrophilic interaction chromatography principle. Bioanalysis. 2020;12:75-85. 10.4155/bio-2019-0188.

7. Eisenhauer EA, Therasse P, Bogaerts J, et al. New response evaluation criteria in solid tumours: revised RECIST guideline (version 1.1). Eur J Cancer. 2009;45(2):228-247. doi:10.1016/j.ejca.2008.10.026. PMID: 19097774

8. Lee JL, Kim MK, Park I, et al. Randomized phase II trial of Sunitinib four weeks on and two weeks off versus two weeks on and one week off in metastatic clear-cell type renal cell carcinoma: RESTORE trial. Ann Oncol. 2015;26:2300-2305.

9. Noda S, Otsuji T, Baba M, et al. Assessment of Sunitinib-Induced Toxicities and Clinical Outcomes Based on Therapeutic Drug Monitoring of Sunitinib for Patients With Renal Cell Carcinoma. Clin Genitourin Cancer. 2015;13(4):350-358. doi:10.1016/j.clgc.2015.01.007. PMID: 25701374.

10. Uemura H, Shinohara N, Yuasa T, et al. A phase II study of sunitinib in Japanese patients with metastatic renal cell carcinoma: insights into the treatment, efficacy and safety. Jpn J Clin Oncol. 2010;40(3):194-202. doi:10.1093/jjco/hyp146. PMID: 19897852.

11. Sehdev S. Sunitinib toxicity management - a practical approach. Can Urol Assoc J. 2016;10(1112Suppl7):S248-S251. doi:10.5489/cuaj.4296

Figure Legends:Figure 1: (A) ROC curve of TTL of sunitinib for the prediction of grade [?] 3 toxicity in all patients. (B) ROC curve of TTL of sunitinib for the prediction of responders in all patients. (C) Kaplan-Meier survival curves illustrating the progression-free survival based on TTL in all patients. Figure 2: (A) ROC curve of toxicity and TTL of sunitinib for the prediction of grade [?] 3 toxicity in patients treated with $2 / 1$ regimen. (B) ROC curve of TTL of sunitinib for the prediction of responders in patients treated with 2/1 regimen. (C) Kaplan-Meier survival curves illustrating the PFS based on TTL in patients treated with $2 / 1$ regimen.

Supplementary Figure 1: Patient flow diagram

Supplementary Figure 2: Box and whisker plots showing trough levels of sunitinib, SU12662 and total sunitinib at steady state $(\mathrm{N}=60)$. The samples for sunitinib and SU12662 analysis was collected between days 10 to 14 in the first cycle of treatment. Supplementary Figure 3: Waterfall plot of best response in tumour size from baseline is shown. 8/12 (66.66\%) patients who had TTL of sunitinib $<60 \mathrm{ng} / \mathrm{mL}$ had PD whereas 4/12 (33.33\%) patients had SD. Among 18 patients who achieved TTL [?] $82.3 \mathrm{ng} / \mathrm{ml}, 2$ (11.11\%) had PD, 13 (72.22\%) patients had SD and three (16.66\%) patients had PR. Out of 16 patients who had TTL of sunitinib in range of $60.75-82.3 \mathrm{ng} / \mathrm{mL}$, one $(6.25 \%)$ patient had $\mathrm{PD}$, fourteen (87.5\%) patients had SD while one patient $(6.25 \%)$ had PR.

\section{Hosted file}


Table 1.docx available at https://authorea.com/users/445085/articles/544702-feasibility-oftherapeutic-drug-monitoring-of-sunitinib-and-its-implications-on-response-and-toxicityin-patients-with-metastatic-renal-cell-cancer

\section{Hosted file}

Figure 1.docx available at https://authorea.com/users/445085/articles/544702-feasibility-oftherapeutic-drug-monitoring-of-sunitinib-and-its-implications-on-response-and-toxicityin-patients-with-metastatic-renal-cell-cancer

\section{Hosted file}

Figure 2.docx available at https://authorea.com/users/445085/articles/544702-feasibility-oftherapeutic-drug-monitoring-of-sunitinib-and-its-implications-on-response-and-toxicityin-patients-with-metastatic-renal-cell-cancer 